\title{
Síntese e caracterização de hidróxidos e óxidos mistos Fe(III):Al com potencial aplicação como pigmentos obtidos a partir da mistura dos géis de goethita e boehmita
}

\author{
(Synthesis and characterization of mixed Fe(III):Al \\ hydroxides and oxides with potential application as pigments \\ obtained from the mixture of goethite and boehmite gels)
}

\author{
E. L. Camenarl, M.A.Bach ${ }^{1}$, R. M. de Carvalho Jr. ${ }^{2}$, R. Marangoni ${ }^{2}$, L. Zatta $^{1 *}$ \\ ${ }^{1}$ Universidade Tecnológica Federal do Paraná, Departamento de Química, Via do conhecimento km 1, \\ 85503-390, Pato Branco, PR, Brasil \\ ${ }^{2}$ Universidade Estadual do Centro-Oeste, Departamento de Química, Guarapuava, PR, Brasil
}

\begin{abstract}
Resumo
Este trabalho propõe uma modificação de metodologia conhecida na literatura baseada no processo sol-gel para a obtenção de hidróxidos e óxidos mistos de $\mathrm{Fe}^{3+} \mathrm{e} \mathrm{Al}^{3+}$. $\mathrm{O}$ método consistiu na mistura dos géis das fases dos hidróxidos precursores obtidos em diferentes condições de $\mathrm{pH}$ e razões $\mathrm{Fe}: \mathrm{Al}$. Os materiais foram caracterizados estruturalmente por difratometria de raios $\mathrm{X}$ (DRX) e espectroscopia vibracional na região do infravermelho por transformada de Fourier (IVTF), quanto à composição pela espectroscopia de energia dispersiva e em relação às propriedades ópticas dos materiais foi empregada a técnica de colorimetria. De maneira geral os hidróxidos mistos tiveram maior cristalinidade quando da mistura dos géis a uma razão molar $\mathrm{Fe} / \mathrm{Fe}+\mathrm{Al}$ de 0,6. Foi observada a influência dos teores de $\mathrm{Al}$ adicionados na estruturação da fase óxido (hematita), onde possivelmente ocorreram substituições isomórficas de $\mathrm{Fe}^{3+}$ por $\mathrm{Al}^{3+}$; tais substituições foram identificadas pelas técnicas de DRX e IVTF. As propriedades observadas pelas diferentes técnicas refletiram nas cores dos materiais obtidos, onde se verificou diferentes colorações dependendo da estrutura cristalina e teor de alumínio nos materiais. Os materiais obtidos pela metodologia empregada apresentam baixo custo e têm potencial de serem aplicados como pigmentos na indústria de tintas.
\end{abstract}

Palavras-chave: hidróxidos mistos, óxidos mistos, colorimetria, pigmentos cerâmicos.

\begin{abstract}
This work proposes a methodology modification on the sol-gel based process reported in the literature to obtain mixed hydroxides and oxides of $\mathrm{Fe}^{3+}$ and $\mathrm{Al}^{3+}$. The method was based on the mixture of the hydroxide gels precursors obtained under different conditions of $\mathrm{pH}$ and $\mathrm{Fe}: \mathrm{Al}$ ratios. The materials were characterized by X-ray diffractometry (XRD), Fourier-transform infrared spectroscopy (FTIR), energy dispersive spectroscopy, and in relation to the optical properties by colorimetric technique. In general, the mixed hydroxides had higher crystallinity when mixing the gels at a Fe/Fe+Al molar ratio of 0.6. It was observed the influence of the Al content added in the structuring of the oxide phase, where the isomorphic substitutions of Fe ${ }^{3+}$ by Al $l^{3+}$ occurred; these substitutions were identified by XRD and FTIR. The characteristics observed by these techniques reflected in the color properties of the obtained materials, where different colorations were observed depending on the crystalline structure and the aluminum content in the materials. The materials obtained by the methodology employed are low cost and possible to be applied as pigments in the paint industry.
\end{abstract}

Keywords: mixed hydroxides, mixed oxides, colorimetry, ceramic pigments.

\section{INTRODUÇÃO}

De maneira geral, hidróxidos mistos baseados em cátions trivalentes como o $\mathrm{Fe}^{3+} \mathrm{e} \mathrm{Al}^{3+}$ podem ser facilmente sintetizados em laboratório tendo como principal finalidade a criação e aplicação de novos compostos obtidos por diferentes metodologias [1]. Os hidróxidos são considerados eficazes quando utilizados como precursores na obtenção de

*leandrozatta@utfpr.edu.br óxidos, devido à sua versatilidade e propriedades estruturais [2]. O óxido de ferro possui algumas características peculiares, dentre as quais destacam-se principalmente suas propriedades químicas, composição e sua natureza mineralógica, possuindo diferentes polimorfos, como as fases $\alpha, \gamma, \varepsilon$, hematita, maghemita, entre outras fases hidratadas. Esta variedade de estruturas possibilita a obtenção de diferentes óxidos de ferro misturados a óxidos de outros elementos que possuem unidades estruturais semelhantes e/ ou compatíveis com os diferentes polimorfos de óxido de 
ferro, melhorando ou gerando novas propriedades a esses materiais. Devido à sua ampla aplicação, óxidos de ferro têm grande importância industrial, sendo fonte de diversas pesquisas [3].

A compatibilidade e a possibilidade de formação de hidróxidos e óxidos mistos de ferro e alumínio se devem aos perfis de cristalização similares, sendo suas estruturas cristalinas formadas por anéis hexagonais de octaedros dos cátions $\mathrm{Fe}^{3+}$ e $\mathrm{Al}^{3+}$, o que possibilita a ocorrência de substituições isomórficas durante a construção dos cristais [4]. Muitos óxidos de ferro possuem estados isoestruturais equivalentes, o que possibilita a ocorrência de substituições isomórficas, alterando principalmente o tamanho da célula unitária. Para que ocorram substituições isomórficas nas estruturas baseadas em óxidos de ferro são necessárias similaridades entre a valência dos cátions, $\mathrm{M}(\mathrm{III})$, e o raio iônico deve ter no máximo uma tolerância de $18 \%$ (menor ou maior) que o raio iônico do ferro hexacoordenado. Por sua vez, o alumínio apresenta as características necessárias, possibilitando substituições isomórficas em sistemas Fe-Al-O [5]. Óxidos baseados em cátions $\mathrm{Fe}^{3+}$ são responsáveis pela cor avermelhada em muitas pedras e solos. Variações na cristalinidade, forma e tamanho de partícula, agregação e substituições isomórficas podem causar variações de cor em minerais de ferro. Todos os óxidos de ferro possuem poder de tingimento, propriedade esta que os permite serem aplicados como pigmentos coloridos [5]. Óxidos de ferro possuem características como resistência a ataques ácidos, básico e à luz do sol, propriedades que os classificam como bons pigmentos. Os óxidos de ferro, além de apresentarem boa proteção da radiação UV, podem evitar processos de degradação química e física em superfícies metálicas. O óxido de alumínio, em especial a alumina, apresenta propriedades distintas às dos óxidos de ferro, como menor resistência a ataques ácidos, porém quando combinado com óxidos de ferro proporcionam a formação de um novo óxido (misto) com propriedades diferentes às de seus precursores, compatíveis com sua aplicação como pigmento [6].

Um método muito utilizado para a obtenção de hidróxidos/ óxidos mistos de ferro e alumínio é o de precipitação [7]; outro método amplamente explorado é o processo sol-gel [8]; alguns trabalhos atuais na literatura reportam a aplicação deste método em processos em que soluções dos metais são misturadas, ou os sais são dissolvidos simultaneamente, e submetidas à adição de hidróxidos de metais alcalinos até o pH desejado [9]. Industrialmente, pigmentos vermelhos de ferro são obtidos a partir transformações no estado sólido pela decomposição térmica de minérios de ferro, em alta pressão, havendo um alto custo e dificuldades no controle de propriedades fundamentais para a obtenção de pigmentos de qualidade [5]. O método sol-gel, juntamente com a síntese hidrotérmica, se destaca para a obtenção de pigmentos de tinta. Os materiais são obtidos em temperaturas baixas e possibilitam um rigoroso controle de propriedades, como pureza, tamanho de partícula e morfologia; estas propriedades influenciam diretamente na cor e aplicabilidade de pigmentos, além de apresentarem baixo custo e serem obtidos por um método considerado ambientalmente correto [6, 10,11]. Este trabalho teve como objetivo avaliar a obtenção de hidróxidos/óxidos mistos de ferro(III) e alumínio por uma metodologia modificada a partir de procedimentos relatados na literatura. Foram avaliadas as condições de síntese dos materiais, como a proporção $\mathrm{Fe}: \mathrm{Al}$ e valor de $\mathrm{pH}$. O método proposto é baseado no processo sol-gel e se diferencia dos relatados na literatura por consistir inicialmente da obtenção de fases estáveis dos hidróxidos de ferro e alumínio separadamente, seguida da mistura dessas, obtendo-se novas fases de hidróxidos mistos dos metais. Os hidróxidos foram calcinados para a obtenção de fases de óxido.

\section{EXPERIMENTAL}

Materiais: os precursores hidróxidos foram sintetizados a partir dos sulfatos de ferro III $\left[\mathrm{Fe}_{2}\left(\mathrm{SO}_{4}\right)_{3} .5 \mathrm{H}_{2} \mathrm{O}\right.$, Vetec, $100 \%$ ] e de alumínio $\left[\mathrm{Al}_{2}\left(\mathrm{SO}_{4}\right)_{3} \cdot 16 \mathrm{H}_{2} \mathrm{O}\right.$, Vetec, $98 \%$ ], com a adição de hidróxido de sódio ( $\mathrm{NaOH}$, Vetec, $97 \%)$.

Síntese dos materiais: os hidróxidos foram sintetizados pelo processo sol-gel, onde inicialmente pesaram-se os sulfatos de ferro e alumínio e prepararam-se separadamente as soluções aquosas destes sais, as quais foram mantidas sob aquecimento $\left(80^{\circ} \mathrm{C}\right)$ e agitação.A cada solução foi adicionado hidróxido de sódio $\left(0,5 \mathrm{~mol} . \mathrm{L}^{-1}, 1\right.$ gota/s) até atingir o $\mathrm{pH}$ desejado (6 ou 8), o qual foi monitorado com o auxílio de um pHmetro (Del Lab, DLA-PH). Após atingida a condição desejada, os géis (hidróxidos) puros foram misturados. Para os casos em que se verificou variação do $\mathrm{pH}$ devido à mistura dos géis, o $\mathrm{pH}$ foi novamente ajustado para o valor de interesse, e as amostras foram mantidas sob agitação e aquecimento por $24 \mathrm{~h}$. Os géis obtidos foram lavados 10 vezes com adição de água destilada (aproximadamente $250 \mathrm{~mL}$ ) seguido de secagem em estufa a $100{ }^{\circ} \mathrm{C}$ por $24 \mathrm{~h}$. Os materiais secos foram macerados com auxílio de gral e pistilo. Para a obtenção das fases de óxido, os hidróxidos obtidos foram calcinados em mufla (Edgcon 3P) a $600{ }^{\circ} \mathrm{C}$ por $3 \mathrm{~h}$. A Tabela I mostra o nome das amostras de hidróxido e óxido, o pH de síntese e as massas dos precursores de sulfato utilizadas em cada amostra.

Caracterizações. Difratometria de raios $X$ (DRX): as análises por DRX das amostras na forma de pó foram realizadas em um difratômetro de bancada (Rigaku, MiniFlex $600)$, utilizando fonte de radiação de cobre $(\mathrm{CuK} \alpha, 1,5418$ A) operado com tensão de $40 \mathrm{kV}$ e corrente de $15 \mathrm{~mA}$, com varredura de $4^{\circ} \cdot \mathrm{min}^{-1} \mathrm{e}$ passo de $0,02^{\circ}$. Espectroscopia no infravermelho com transformada de Fourier (IVTF): os espectros de infravermelho foram obtidos em espectrômetro (Perkin Elmer, Frontier), pelo acúmulo de 32 varreduras no intervalo de $4000 \mathrm{a} 400 \mathrm{~cm}^{-1} \mathrm{e}$ resolução de $4 \mathrm{~cm}^{-1}$. As análises foram realizadas utilizando pastilhas de $\mathrm{KBr}$ contendo $1 \%$ em massa das amostras diluídas. Todas as amostras foram diluídas na mesma concentração para comparações de intensidades das bandas nos espectros. Espectroscopia de energia dispersiva $(E D S)$ : análises químicas por EDS 
Tabela I - Identificação das amostras e condições de síntese.

[Table I - Identification of the sample and conditions of synthesis.]

\begin{tabular}{cccccccc}
\hline \multicolumn{2}{c}{ Amostra } & pH de & \multicolumn{2}{c}{ Massa de precursor $(\mathrm{g})$} & \multicolumn{2}{c}{$\mathrm{Mol}\left(10^{-3} \mathrm{~mol}\right)$} & $\mathrm{Fe} / \mathrm{Fe}+\mathrm{Al}$ \\
Hidróxido & Óxido & síntese & $\mathrm{Fe}_{2}\left(\mathrm{SO}_{4}\right)_{3}$ & $\mathrm{Al}_{2}\left(\mathrm{SO}_{4}\right)_{3}$ & $\mathrm{Fe}^{3+}$ & $\mathrm{Al}^{3+}$ & adicionado \\
\hline $\mathrm{A}$ & $\mathrm{K}$ & & 2,00 & 0,00 & 3,6 & 0 & 1,0 \\
$\mathrm{~B}$ & $\mathrm{~L}$ & & 2,00 & 0,80 & 3,6 & 1,6 & 0,7 \\
$\mathrm{C}$ & $\mathrm{M}$ & \multirow{2}{*}{6} & 2,00 & 1,30 & 3,6 & 2,6 & 0,6 \\
$\mathrm{D}$ & $\mathrm{N}$ & & 2,00 & 1,80 & 3,6 & 3,6 & 0,5 \\
$\mathrm{E}$ & $\mathrm{O}$ & & 0 & 2,60 & 0 & 5,2 & 0 \\
\hline $\mathrm{F}$ & $\mathrm{P}$ & & 2,00 & 0,00 & 3,6 & 0 & 1,0 \\
$\mathrm{G}$ & $\mathrm{Q}$ & & 2,00 & 0,80 & 3,6 & 1,6 & 0,7 \\
$\mathrm{H}$ & $\mathrm{R}$ & \multirow{2}{*}{8} & 2,00 & 1,30 & 3,6 & 2,6 & 0,6 \\
$\mathrm{I}$ & $\mathrm{S}$ & & 2,00 & 1,80 & 3,6 & 3,6 & 0,5 \\
$\mathrm{~J}$ & $\mathrm{~T}$ & & 0 & 2,60 & 0 & 5,2 & 0 \\
\hline
\end{tabular}

foram realizadas utilizando-se microscópio eletrônico de varredura (MEV, Hitachi, TM3000) com detector (EDS) SwiftED3000 da Bruker acoplado, operado a $15 \mathrm{kV}$ e fonte de filamento de tungstênio com magnificação de 500 vezes. As amostras foram depositadas no stub de análise na forma de pó e prensadas com uma espátula sobre fita de carbono. Os resultados foram expressados em porcentagem atômica dos elementos presentes nas amostras. Análise térmica diferencial e termogravimétrica (DTA-TGA): as análises de decomposição térmica dos hidróxidos obtidos foram realizadas em um equipamento T.A. Instruments, SDT Q-600, sendo utilizados cadinhos de alumina e faixa de leitura de 30-1100 ${ }^{\circ} \mathrm{C}$, com rampa de aquecimento de 10 ${ }^{\circ} \mathrm{C}$. $\mathrm{min}^{-1}$ sob atmosfera de ar sintético com fluxo constante de $30 \mathrm{~mL} \cdot \mathrm{min}^{-1}$. Colorimetria: a análise colorimétrica dos materiais foi realizada em um colorímetro portátil (Konica Minolta, CR 400), equipado com câmara escura para evitar possíveis interferências nas leituras e o sistema CIELAB foi adotado para interpretações e posterior discussão. Foram determinados os valores da diferença de cor pela Eq. A:

$$
\Delta \mathrm{Eab}=\left[(\Delta \mathrm{L})^{2}+(\Delta \mathrm{a})^{2}+(\Delta \mathrm{b})^{2}\right]^{1 / 2}
$$

onde, $\Delta \mathrm{E}$ é a diferença total de cor, $\Delta \mathrm{L}$ a diferença de luminosidade, $\Delta \mathrm{a}$ a diferença entre os tons vermelho e verde e $\Delta \mathrm{b}$ a diferença entre tons amarelo e azul [12].

\section{RESULTADOS E DISCUSSÃO}

Na Fig. 1 são mostrados os difratogramas de raios $X$ das amostras de hidróxidos e óxidos obtidos. Em alguns difratogramas não são mostradas as regiões de 5 a $10^{\circ}(2 \theta)$ devido à ausência de picos. Analisando-se os difratogramas dos hidróxidos puros de ferro (amostras A e F) e alumínio (amostras E e J), mostrados nas Figs. 1a e 1b, verificou-se que foram obtidas fases cristalinas de goethita $[\mathrm{FeO}(\mathrm{OH})]$ e boehmita $[\mathrm{AlO}(\mathrm{OH})]$, indicadas nos difratogramas pelas letras $\mathrm{G}$ e $\mathrm{B}$, respectivamente. A identificação destas fases foi em acordo com as análises cristalográficas realizadas no software PDXI 2.0 e arquivos cristalográficos ICDD01-074-2195 para a goethita e ICDD-01-088-2110 para a boehmita. Vale destacar a amostra F, para o hidróxido de ferro puro obtido em $\mathrm{pH} 8$, onde não se obteve fase cristalina do mesmo; esse comportamento já era esperado, pois hidróxidos de ferro obtidos em condições semelhantes e relatados na literatura apresentaram padrão de difração de materiais amorfos [13]. É conhecido que a goethita passa a ter uma solubilidade considerável em $\mathrm{pH}$ acima de 8 , o que pode ter contribuído para que a amostra $\mathrm{F}$ tenha se mostrado amorfa [5]. Considerando ainda o efeito do $\mathrm{pH}$ na síntese dos hidróxidos puros, verificou-se que para o alumínio o aumento do $\mathrm{pH}$ contribuiu para uma maior organização estrutural, pois os picos característicos da boehmita na amostra $\mathbf{J}$ se apresentaram relativamente mais finos e melhores definidos em comparação à amostra obtida em pH 6 (amostra E).

Para os hidróxidos mistos obtidos em pH 6 (Fig. 1a), verificou-se que quando da mistura dos géis precursores na razão $\mathrm{Fe} / \mathrm{Fe}+\mathrm{Al}$ de 0,7 a estrutura da goethita foi destruída (amostra B), porém foi observado um pico largo na região de $35^{\circ}(2 \theta)$, o qual não se refere à estrutura da goethita e nem da boehmita (amostras A e E), indicando a possível formação de uma nova fase. Quando se aumentou o teor de alumínio (teor $\mathrm{Fe} / \mathrm{Fe}+\mathrm{Al}$ de 0,6 , amostra $\mathrm{C}$ ), verificaram-se picos definidos e de intensidade considerável, característicos para a fase goethita, e picos largos de baixa intensidade da fase boehmita; o mesmo comportamento foi verificado para amostra com teor $\mathrm{Fe} / \mathrm{Fe}+\mathrm{Al}$ de 0,5 (amostra D). Para estas duas amostras verificou-se que o pico largo na região de $35^{\circ}(2 \theta)$ tornou-se mais intenso de acordo com o aumento no teor de Al, indicando a possível formação de uma fase mista de hidróxido de ferro e alumínio, baseado na estrutura cristalina da goethita, devido a substituições isomórficas de ferro por alumínio. Para as amostras de hidróxidos mistos obtidos em pH 8 (Fig. 1 b), verificou-se de maneira geral que após a mistura dos géis a fase amorfa do precursor de hidróxido de ferro (amostra F) passou a apresentar maior 

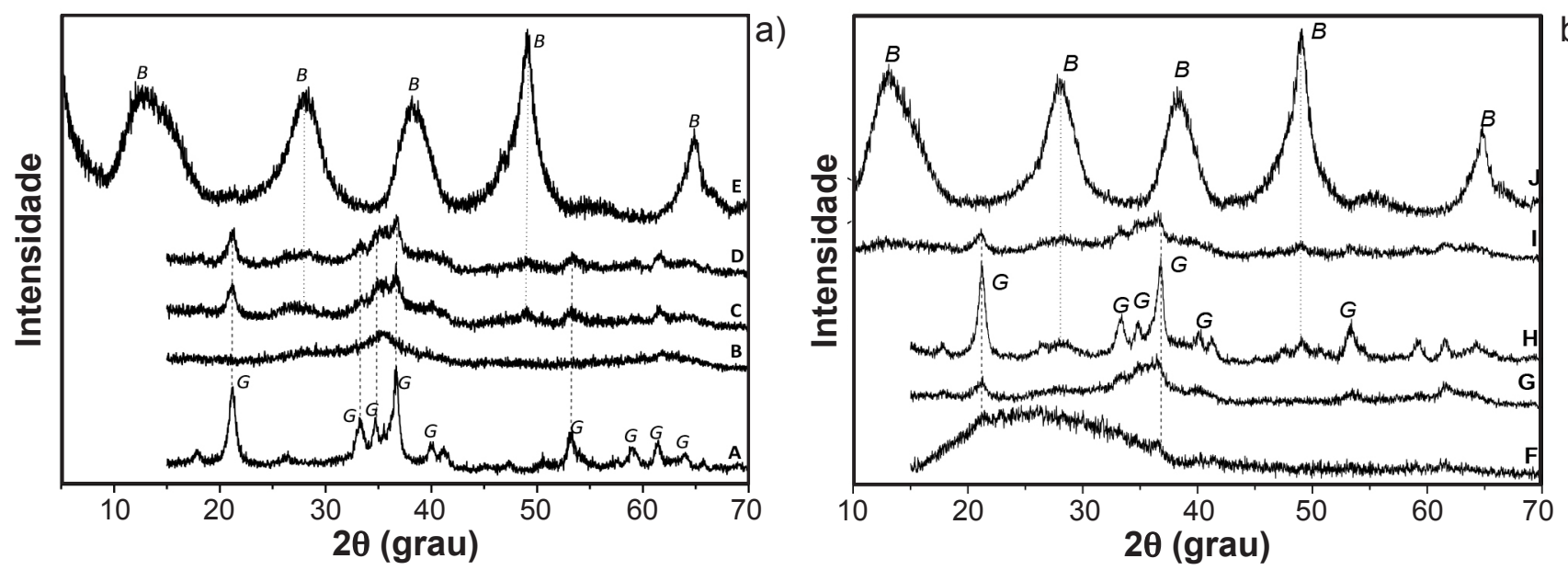

b)
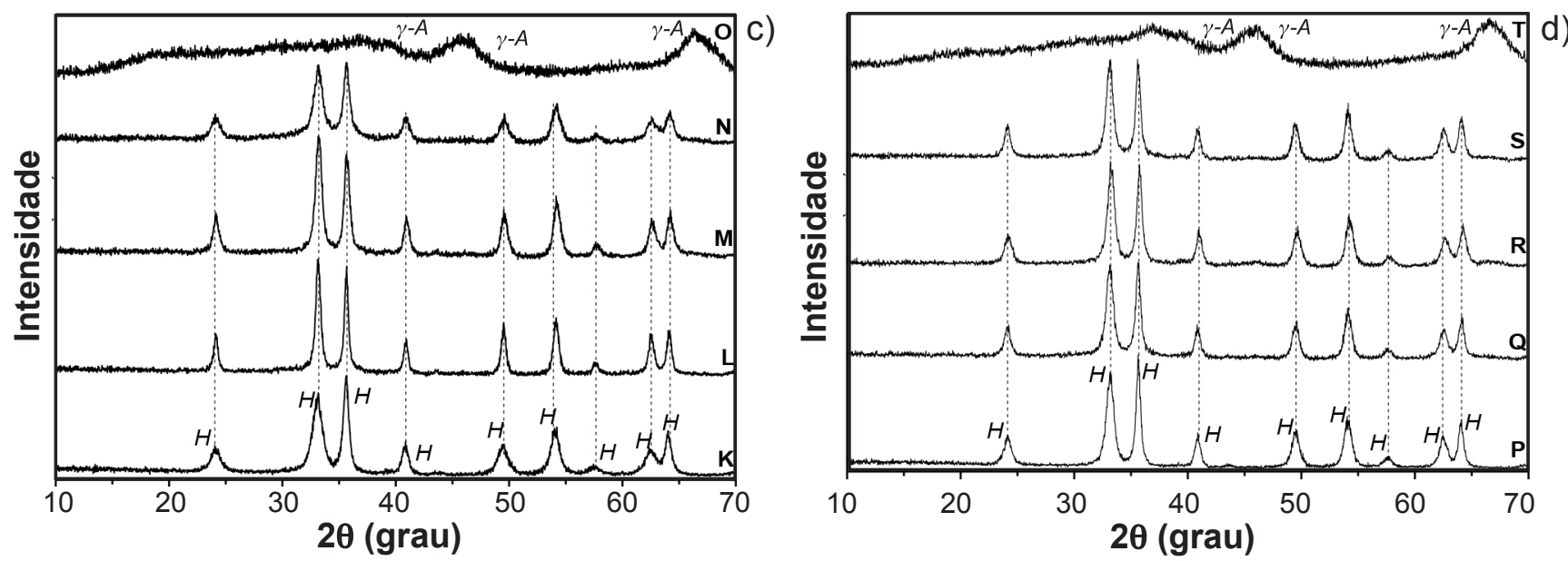

Figura 1: Difratogramas de raios X para os hidróxidos obtidos em pH 6 (a) e pH 8 (b) e óxidos em pH 6 (c) e pH 8 (d). B - boehmita; $\mathrm{G}$ - goethita; $\boldsymbol{\gamma}$-A - $\boldsymbol{\gamma}$-alumina; $\mathrm{H}$ - hematita.

[Figure 1: X-ray diffraction patterns for hydroxides obtained in pH 6 (a) and pH 8 (b), and oxides in pH 6 (c) and pH 8 (d). B - boehmite; $G$ - goethite; $\boldsymbol{\gamma}$ - - $\boldsymbol{\gamma}$-alumina; $H$ - hematite.]

grau de cristalinidade, pois surgiram picos característicos da goethita devido à adição do hidróxido de alumínio. A amostra contendo a razão $\mathrm{Fe} / \mathrm{Fe}+\mathrm{Al}$ de 0,6 (amostra $\mathrm{H}$ ) se destacou entre todas as amostras de hidróxido por apresentar picos característicos da goethita melhores definidos e mais intensos. O mesmo comportamento foi observado para a amostra sintetizada em pH 6 (amostra C, Fig. 1a). As amostras obtidas nas razões $\mathrm{Fe} / \mathrm{Fe}+\mathrm{Al}$ 0,7 e 0,5, amostras $\mathrm{G}$ e I, respectivamente, além de apresentarem picos de baixa intensidade característicos da goethita, apresentaram um pico largo na região de $35^{\circ}(2 \theta)$.

As Figs. 1c e 1d mostram os difratogramas de raios $\mathrm{X}$ dos óxidos obtidos a partir da calcinação dos hidróxidos sintetizados nas duas faixas de $\mathrm{pH}$ avaliadas. Verificaramse nos difratogramas dos óxidos de ferro e alumínio puros, amostras $\mathrm{K}, \mathrm{O}, \mathrm{P}$ e $\mathrm{T}$, picos característicos das fases hematita $\left(\mathrm{Fe}_{2} \mathrm{O}_{3}\right.$, ICDD-01-072-0469) e fase alumina $\left(\gamma-\mathrm{Al}_{2} \mathrm{O}_{3}\right.$, JCPDS 10-0425). Os três picos indicados por $\gamma$-A nos difratogramas se referem aos planos cristalográficos (311), (400) e (440). As fases dos óxidos de ferro puros se mostraram bem cristalinas com picos característicos finos e intensos, enquanto que os óxidos de alumínio possuíram baixa cristalinidade apresentando picos característicos largos. Isso se deve possivelmente ao fato de que a temperatura de calcinação empregada neste trabalho foi abaixo da temperatura de obtenção de fases altamente cristalinas do óxido de alumínio [5]. Observou-se nos difratogramas das amostras dos óxidos mistos, nas duas faixas de $\mathrm{pH}$ avaliadas, que o pico característico da hematita em $35,7^{\circ}(2 \theta)$ diminuiu de intensidade em relação ao pico em $33,7^{\circ}(2 \theta)$ conforme se aumentou o teor de alumínio adicionado; este comportamento se destacou nas amostras com razões $\mathrm{Fe} / \mathrm{Fe}+\mathrm{Al}$ 0,7 e 0,6 (amostras L, M, Q e R). Esta observação indicou que a presença do alumínio interfere na estruturação da fase hematita nas condições de síntese empregadas. Como observado de maneira geral por DRX (Fig. 1), foram obtidas estruturas dos hidróxidos baseadas na fase goethita; este polimorfo do hidróxido de ferro com substituições isomórficas do $\mathrm{Fe}$ pelo $\mathrm{Al}$ é o mais estudado na literatura, sendo que esse fenômeno acontece amplamente em amostras naturais. As substituições isomórficas ocorrem porque o cátion $\mathrm{Al}^{3+}$ é $17 \%$ menor que a espécie $\mathrm{Fe}^{3+}$, sendo possíveis substituições de até $1 / 3$ de ferro por alumínio [5].

Não foram observados nos óxidos mistos picos de 
intensidade considerável, característicos da fase alumina e/ ou picos indicando espécies derivadas do alumínio, sendo que fases baseadas na alumina foram obtidas apenas nas amostras obtidas a partir do hidróxido de alumínio puro. Com os dados obtidos, acredita-se que ocorreram substituições isomórficas de espécies de ferro por alumínio. Além disso, observou-se que houve deslocamentos em alguns picos de difração característicos da hematita com o aumento da concentração do alumínio na estrutura cristalina. A hematita é um mineral que apresenta planos cristalinos em $\sim 24^{\circ}(012)$ e $\sim 49^{\circ}$ (024) [9]; para avaliar os deslocamentos dos picos nos difratogramas, a região próxima a $49^{\circ}(2 \theta)$ foi ampliada (não mostrada) e a distância d do plano (024) foi calculada pela lei de Bragg; os resultados são mostrados na Tabela II juntamente com dados de parâmetros de rede e volume de célula unitária (dados determinados pelo software PDXI 2.0). Observou-se de maneira geral que os fatores avaliados variaram com o aumento da concentração de $\mathrm{Al}^{3+}$, os quais refletiram na distância do plano (024) e na diminuição dos parâmetros de rede, mesmo para a razão $\mathrm{Fe} / \mathrm{Fe}+\mathrm{Al}$ 0,6 nos dois valores de $\mathrm{pH}$ avaliados, amostras $\mathrm{M}$ e R. Quando da mistura na razão $\mathrm{Fe} / \mathrm{Fe}+\mathrm{Al}$ 0,5 (amostras $\mathrm{N}$ e $\mathrm{S}$ ), houve aumento dos valores dos parâmetros. Pelo fato de que os menores valores dos parâmetros foram encontrados nas amostras obtidas na proporção $\mathrm{Fe} / \mathrm{Fe}+\mathrm{Al}$ 0,6 (amostras $\mathrm{M}$ e R), independente do $\mathrm{pH}$ de síntese, acredita-se que nesta proporção ocorreu o máximo grau de substituições isomórficas nas condições experimentais empregadas e os resultados estão de acordo com relatos na literatura que demonstram haver um limite de substituições de sítios de $\mathrm{Fe}^{3+}$ por $\mathrm{Al}^{3+}[5]$.

Nas Figs. 2a e 2b são mostrados os perfis de decomposição térmica a partir das curvas termogravimétricas (TGA), enquanto que nas Figs. 2c e 2d são mostradas as curvas de análise térmica diferencial (DTA) dos hidróxidos. A Tabela III mostra as temperaturas dos eventos térmicos (dados das curvas de DTA). Analisando-se os dados para as amostras de hidróxidos mistos, observou-se que apenas quando da

Tabela II - Parâmetros de rede e volume de cela das amostras de óxidos de ferro e óxidos mistos de ferro e alumínio.

[Table II - Lattice parameters and cell volume of samples of iron oxides and mixed oxides of iron and aluminum.]

\begin{tabular}{ccccc}
\hline Amostra & $\mathrm{d}_{(024)}(\AA)$ & $\mathrm{a}(\AA)$ & $\mathrm{c}(\AA)$ & $\mathrm{V}\left(\AA^{3}\right)$ \\
\hline $\mathrm{K}$ & 7,367 & 5,038 & 13,791 & 303,15 \\
$\mathrm{~L}$ & 7,363 & 5,030 & 13,770 & 301,71 \\
$\mathrm{M}$ & 7,354 & 5,028 & 13,737 & 300,81 \\
$\mathrm{~N}$ & 7,360 & 5,038 & 13,752 & 302,23 \\
$\mathrm{P}$ & 7,365 & 5,033 & 13,758 & 301,80 \\
$\mathrm{Q}$ & 7,361 & 5,036 & 13,761 & 302,23 \\
$\mathrm{R}$ & 7,345 & 5,020 & 13,729 & 299,57 \\
$\mathrm{~S}$ & 7,360 & 5,035 & 13,756 & 301,96 \\
\hline
\end{tabular}

Nota: os parâmetros das amostras de óxido de alumínio puro não são mostrados (amostras $\mathrm{O}$ e $\mathrm{T}$ ). presença de teores de alumínio nas amostras, ou até mesmo para a amostra de hidróxido de alumínio puro, houve a ocorrência de um evento endotérmico entre 300 e $450{ }^{\circ} \mathrm{C}$; porém, para a amostra de hidróxido de ferro puro, tal evento não ocorreu. Esse evento se deve possivelmente a alguma reação relacionada ao hidróxido de alumínio, como o processo de desidroxilação. Para a temperatura de formação da hematita, verificou-se de maneira geral que quanto mais cristalina a amostra, como observado pelos difratogramas de raios X (Fig. 1), menor foi a temperatura de desidroxilação e os picos endotérmicos nas curvas de DTA (Fig. 3) se apresentaram mais intensos e finos. Outro dado que se destacou se deve ao fato de que a transição do hidróxido de alumínio para fase alumina ocorreu em temperaturas menores quando dos materiais mistos em comparação à obtenção da fase alumina pura. Em relação ao efeito do $\mathrm{pH}$ nas propriedades térmicas dos diferentes hidróxidos, verificou-se que o aumento do $\mathrm{pH}$ de síntese fez com que os eventos térmicos tenderam a ocorrer em temperaturas maiores. Os dados das curvas de TGA e DTA justificaram a escolha da temperatura de calcinação dos materiais em $600{ }^{\circ} \mathrm{C}$, pois nesta, com exceção dos hidróxidos de alumínio puros, a fase hematita foi formada a partir de todos os hidróxidos precursores.

Os espectros obtidos por IVTF das amostras são mostrados nas Figs. 3 e 4, separados em grupos de hidróxidos e óxidos e de acordo com o pH 6 ou 8. Os valores referentes aos números de onda e suas respectivas atribuições são mostrados na Tabela IV. Analisando-se os espectros de IVTF obtidos para os hidróxidos mistos de ferro e alumínio (Fig. 3), observaram-se vibrações relacionadas à água em 3487 e $\sim 1637 \mathrm{~cm}^{-1}$, as quais corresponderam ao estiramento da ligação $\mathrm{O}-\mathrm{H}$ e à vibração de deformação angular da água $\mathrm{H}-\mathrm{O}-\mathrm{H}$, respectivamente $[9,14,15]$. As vibrações nos espectros de IVTF características da fase do hidróxido de ferro(III) se encontraram em $\sim 890$ e $\sim 790 \mathrm{~cm}^{-1}$ para a deformação angular $\mathrm{Fe}-\mathrm{O}-\mathrm{H}$, as quais indicaram a presença de grupamentos de hidroxila estruturais. A vibração em 890 $\mathrm{cm}^{-1}$ se destaca por ser uma banda que caracteriza a goethita $(\alpha-\mathrm{FeOOH})$, exceto nas amostras E e J [15]. A identificação da goethita pela técnica de IVTF pode ser relacionada com os difratogramas de raios $\mathrm{X}$ das amostras, onde esta fase do hidróxido de ferro também foi identificada. Verificouse que as vibrações características da fase goethita estavam presentes em todas as amostras mistas, indicando que essa estrutura se manteve após a mistura dos géis. A vibração em $1065 \mathrm{~cm}^{-1}$ característica da fase boehmita, identificada nos espectros das amostras contendo apenas alumínio, E e J (Figs. 3a e 3b, respectivamente), foi atribuída à vibração $\mathrm{AlOOH}$ [16]. Para as amostras mistas, independente do pH de síntese, verificou-se a presença do pico em 1065 $\mathrm{cm}^{-1}$, a qual possivelmente indica a presença de espécie baseada no hidróxido de alumínio na estrutura da goethita, como verificado por DRX. As vibrações na região entre 980 e $1077 \mathrm{~cm}^{-1}$ são características do íon $\mathrm{SO}_{4}^{2-}$, porém na amostra $\mathrm{B}$ esta banda foi a mais intensa. As presenças destas vibrações indicaram a presença de teores de ânions de sulfato 

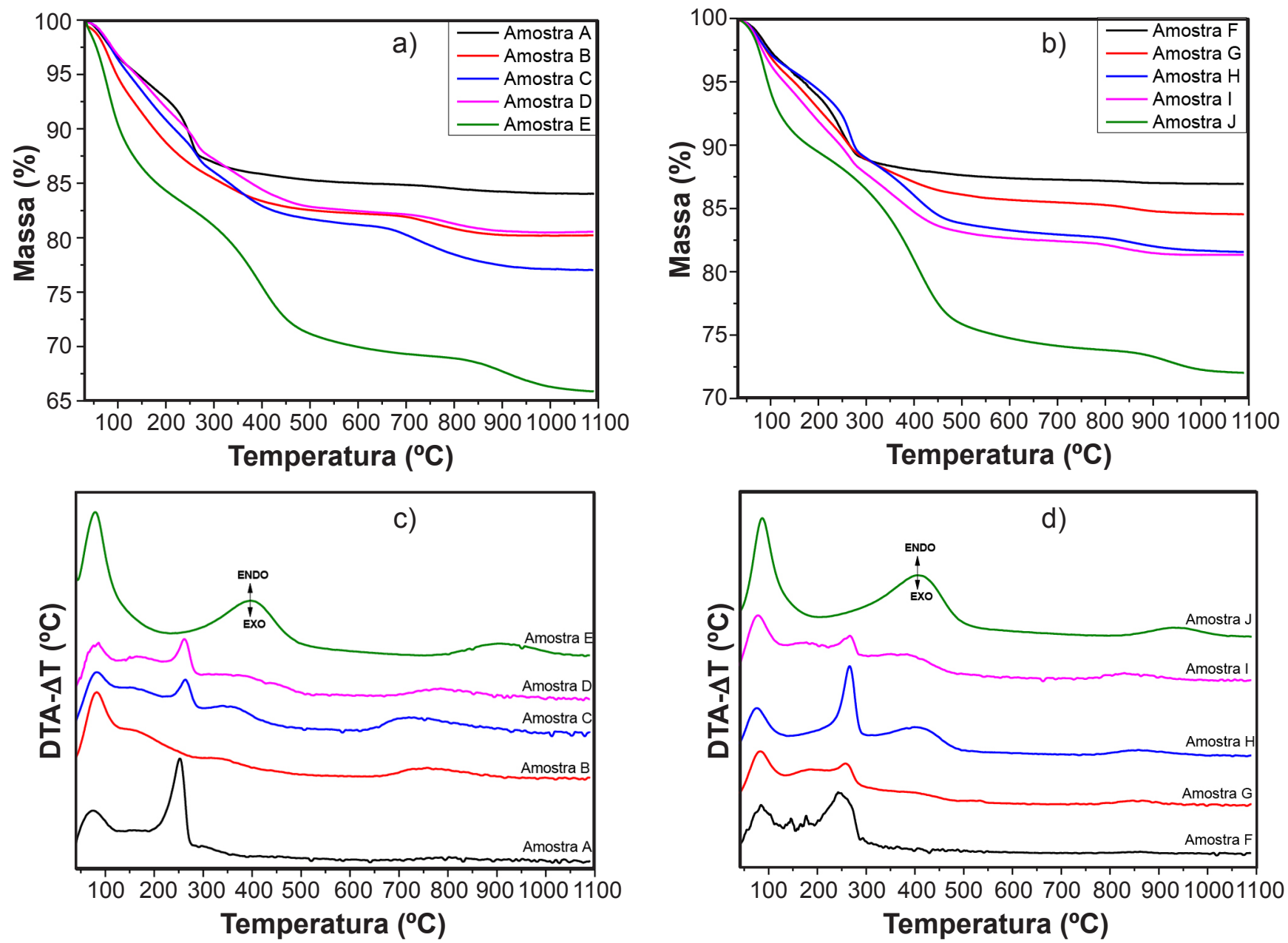

Figura 2: Curvas de análise termogravimétrica (TGA) para os hidróxidos obtidos em pH 6 (a) e pH 8 (b) e suas respectivas curvas de análise térmica diferencial, DTA (c, d).

[Figure 2: Curves of thermal gravimetric analysis (TGA) for the hydroxides obtained in pH $6(a)$ and pH 8 (b), and their respective differential thermal analysis curves, DTA $(c, d)$.]

Tabela III - Identificação dos eventos térmicos nas curvas de DTA dos hidróxidos.

[Table III - Identification of thermal events in the DTA curves of hydroxides.]

\begin{tabular}{ccccccc}
\hline & Amostra & $\begin{array}{c}\text { Água fisissorvida } \\
\left({ }^{\circ} \mathrm{C}\right)\end{array}$ & $\begin{array}{c}\text { Desidratação } \\
\left({ }^{\circ} \mathrm{C}\right)\end{array}$ & $\begin{array}{c}\text { Formação de } \\
\text { hematita }\left({ }^{\circ} \mathrm{C}\right)\end{array}$ & $\begin{array}{c}\text { Fase mista } \\
\left({ }^{\circ} \mathrm{C}\right)\end{array}$ & $\begin{array}{c}\text { Formação de } \\
\text { alumina }\left({ }^{\circ} \mathrm{C}\right)\end{array}$ \\
\hline & $\mathrm{A}$ & 75,60 & - & 251,60 & - & - \\
$\mathrm{pH}$ & $\mathrm{B}$ & 83,28 & 177,33 & - & 343,67 & 756,55 \\
6 & $\mathrm{C}$ & 83,28 & 167,45 & 263,47 & 365,21 & 731,07 \\
& $\mathrm{D}$ & 85,26 & 173,38 & 261,49 & 390,70 & 780,04 \\
& $\mathrm{E}$ & 79,33 & - & $*$ & 409,99 & 922,89 \\
\hline & $\mathrm{F}$ & 83,73 & - & 246,19 & - & - \\
$\mathrm{pH}$ & $\mathrm{G}$ & 81,96 & 185,02 & 260,39 & 411,13 & 859,83 \\
8 & $\mathrm{H}$ & 76,47 & 185,03 & 265,89 & 409,15 & 865,32 \\
& $\mathrm{I}$ & 80,21 & 185,02 & 265,89 & 396,40 & 846,87 \\
& $\mathrm{~J}$ & 87,46 & - & $*$ & 405,64 & 946,19 \\
\hline
\end{tabular}

Notas: * o evento não ocorreu devido à ausência de ferro na amostra; - ausência de pico característico.

possivelmente não removidos no processo de lavagem [17].

Comparando-se a definição das bandas nos espectros de IVTF com a cristalinidade dos materiais por DRX, verificou-se para as amostras preparadas em pH 6 (Figs. 

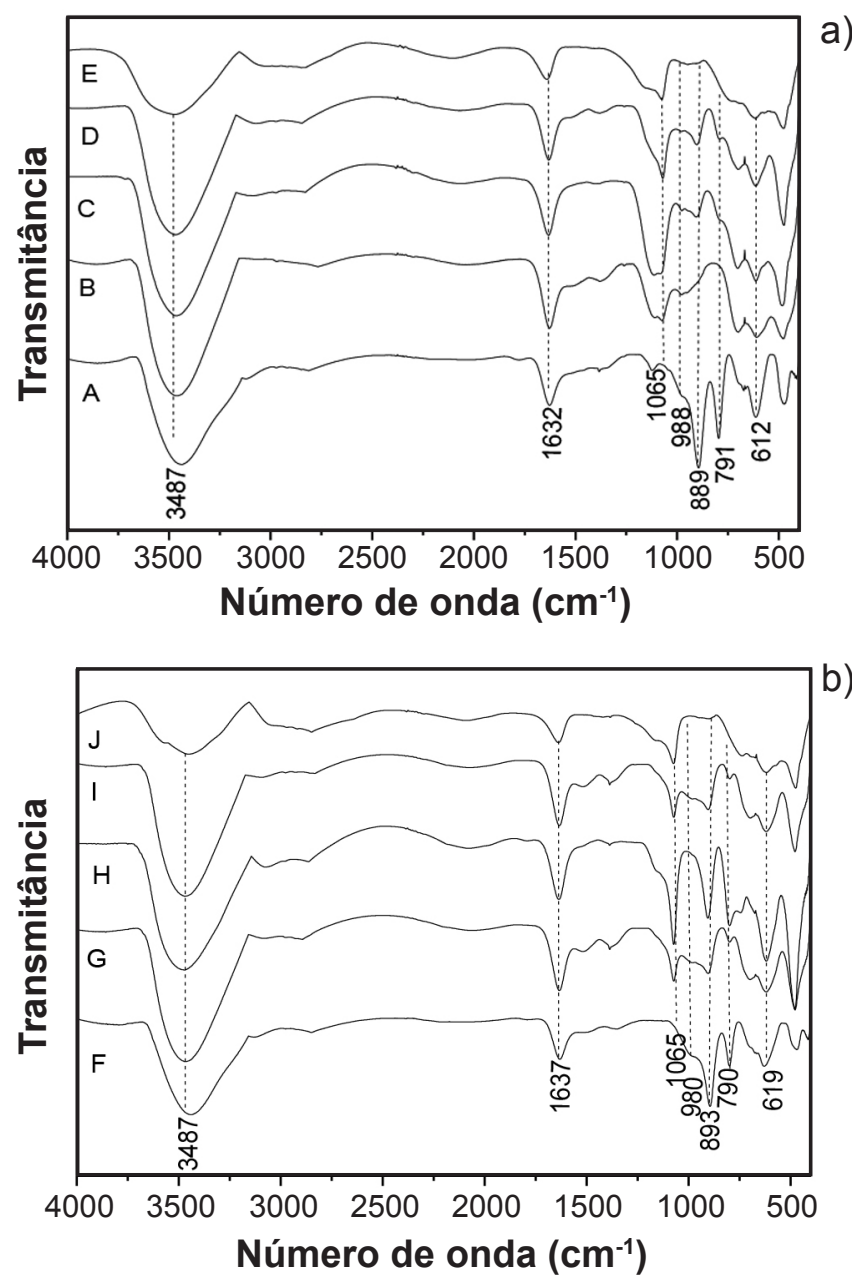

Figura 3: Espectros de IVTF dos hidróxidos mistos de Fe:Al: (a) $\mathrm{pH} \mathrm{6}$; e (b) $\mathrm{pH} 8$.

[Figure 3: IR spectra of the mixed hydroxides of Fe:Al: (a) pH 6; and (b) $\mathrm{pH} 8$.

3a e 1a, respectivamente) que a amostra A apresentou uma banda em aproximadamente $890 \mathrm{~cm}^{-1}$ mais intensa e definida, sendo que esta mesma amostra apresentou picos de difração da fase goethita também mais intensos e definidos em comparação às outras amostras. Ainda analisando a vibração em $890 \mathrm{~cm}^{-1}$, porém para as amostras mistas, verificou-se que esta banda foi mais intensa e melhor definida nos espectros de IVTF naquelas amostras que se mostraram mais cristalinas (DRX); tal observação é melhor destacada para a amostra H. Tais observações indicaram que quanto mais intensa e melhor definida a banda nos espectros de IVTF em $890 \mathrm{~cm}^{-1}$ o material se apresenta mais organizado a curto alcance. Outra banda de vibração nos espectros de IVTF que se relacionou com a organização da fase goethita foi a banda na região de $791 \mathrm{~cm}^{-1}$ para estiramento $\mathrm{Fe}$ $\mathrm{OH}-\mathrm{Fe}$ no plano [18], onde quanto melhor estruturado o material, mais intensa e melhor definida se mostrou esta banda de vibração. Para todos os hidróxidos, os espectros de IVTF apresentaram bandas de vibração na região de 612 e 619 cm$^{-1}$ características de M-O e de flexão M-O-H, onde $\mathrm{M}$ - Fe ou $\mathrm{Al}$ [19]. Comparando-se os espectros das

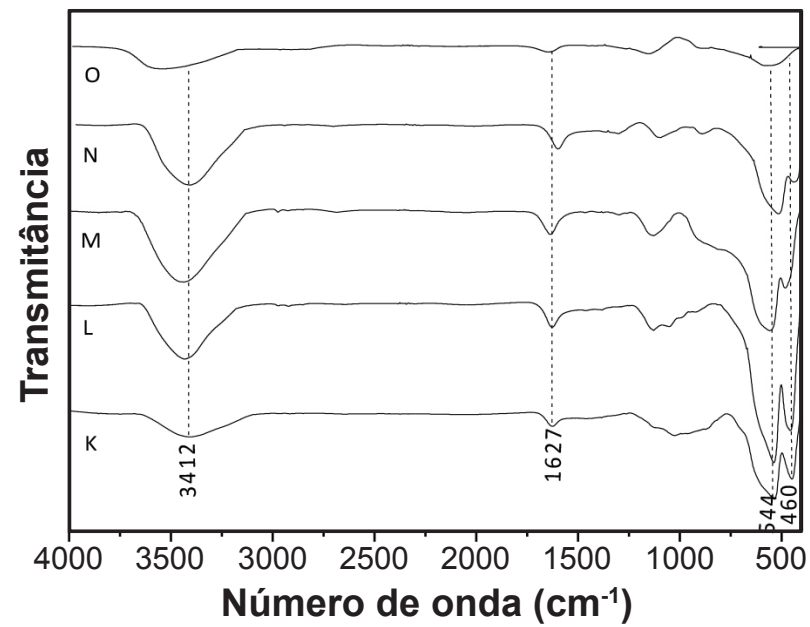

a)

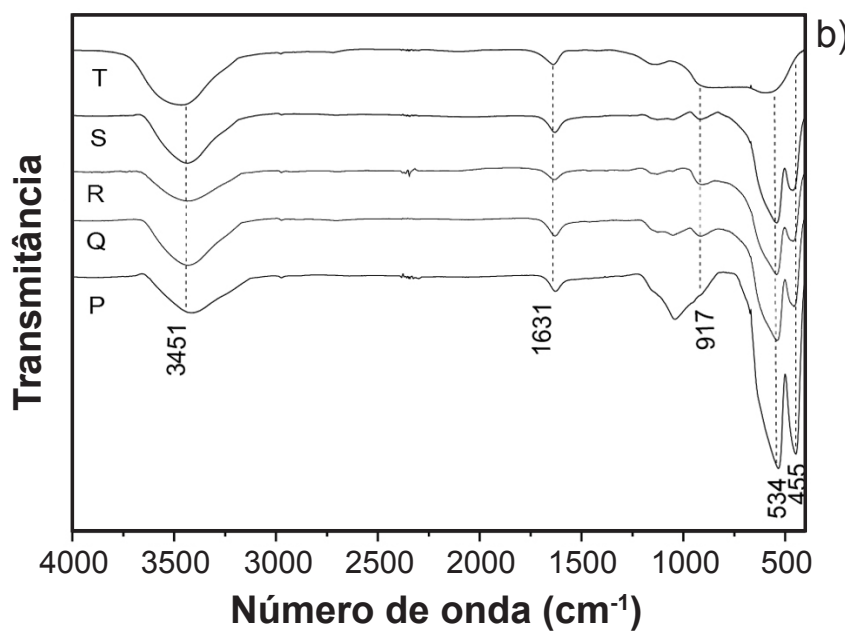

Figura 4: Espectros de IVTF dos óxidos mistos de Fe:Al: (a) $\mathrm{pH}$ 6; e (b) $\mathrm{pH} 8$.

[Figure 4: IR spectra of the mixed oxides of Fe:Al: (a) $\mathrm{pH}$ 6; and (b) $\mathrm{pH} 8$.

amostras de hidróxido obtidas em $\mathrm{pH} 6$ com aquelas em pH 8 verificou-se na região de $\sim 3487 \mathrm{~cm}^{-1}$ que as vibrações se mantiveram para todos os hidróxidos, com pequenas diferenças na intensidade do pico. O pico na região de $1065 \mathrm{~cm}^{-1}$, característico da boehmita, se apresentou mais fino, melhor definido e de maior intensidade nas amostras sintetizadas em $\mathrm{pH} 8$, indicando melhor estruturação da fase de hidróxido de alumínio em $\mathrm{pH}$ maior. Em torno de $980 \mathrm{~cm}^{-1}$ as amostras com maiores teores de alumínio se apresentaram melhores definidas em ambos os pHs de síntese, devido possivelmente à melhor estruturação da fase goethita. Na região de $\sim 890 \mathrm{~cm}^{-1}$ observou-se que com o aumento do teor de alumínio ocorreu a redução da intensidade da vibração, enquanto que no pH 8 a mesma se tornou mais intensa em comparação às amostras obtidas em pH 6. As vibrações entre 612 e $480 \mathrm{~cm}^{-1}$ permaneceram com bandas bem definidas em todos os hidróxidos.

As transformações das fases de hidróxido a óxido foram verificadas pela ausência dos picos referentes às hidroxilas estruturais em $\sim 890$ e $790 \mathrm{~cm}^{-1}$ [15] nos espectros da Fig. 4. As vibrações características da hematita em 460 e $544 \mathrm{~cm}^{-1}$ 
[15] apresentaram deslocamentos quando foram adicionados teores de $\mathrm{Al}$, em comparação às amostras puras de $\mathrm{Fe}$ (amostras A, K, F e P); esses deslocamentos podem indicar a substituição isomórfica do $\mathrm{Fe}$ pelo $\mathrm{Al}$. A região entre 1020 e $915 \mathrm{~cm}^{-1}$ para vibrações características da ligação Al-O [19] também pode indicar a ocorrência de substituições isomórficas. Nas amostras Q, R, S e T essa banda foi observada em $917 \mathrm{~cm}^{-1}$; o fato desta banda ter se destacado

Tabela IV - Vibrações características para os hidróxidos e óxidos mistos de ferro e alumínio.

[Table IV - Characteristic vibrations for the mixed iron and aluminum hydroxides and oxides.]

\begin{tabular}{|c|c|}
\hline $\begin{array}{l}\text { Número de } \\
\text { onda }\left(\mathrm{cm}^{-1}\right)\end{array}$ & Hidróxido misto - atribuição \\
\hline$\sim 3487$ & Estiramento da ligação O-H \\
\hline$\sim 1637$ & Deformação angular da água \\
\hline$\sim 1074$ & Íon $\mathrm{SO}_{4}^{2-}$ \\
\hline$\sim 1065$ & Vibração AlOOH \\
\hline$\sim 790$ e $\sim 890$ & Deformação angular Fe-O-H \\
\hline$\sim 980$ & Íon $\mathrm{SO}_{4}^{2-}$ \\
\hline$\sim 890$ & Goethita $(\alpha-\mathrm{FeOOH})$ \\
\hline$\sim 791$ & Estiramento $\mathrm{Fe}-\mathrm{OH}-\mathrm{Fe}$ \\
\hline \multirow[t]{2}{*}{$\sim 612$} & $\begin{array}{c}\text { Vibrações características a M-O e } \\
\text { de flexão M-O-H }\end{array}$ \\
\hline & Óxido misto - atribuição \\
\hline$\sim 3412$ & Vibrações O-H \\
\hline$\sim 1627$ & Deformação angular da água \\
\hline$\sim 1020$ e $\sim 915$ & Vibrações Al-O \\
\hline$\sim 917$ & Ligações Al-O \\
\hline$\sim 544 \mathrm{e} \sim 460$ & Hematita $-\mathrm{Fe}_{2} \mathrm{O}_{3}$ \\
\hline
\end{tabular}

nas amostras sintetizadas em $\mathrm{pH} 8$ mostra a influência desta variável, indicando que o $\mathrm{pH}$ possivelmente contribui para maiores teores e/ou melhor estruturação dos sítios de alumínio na estrutura da hematita, o que possivelmente refletiu nas propriedades térmicas observadas pelas técnicas de TGA/DTA.

A Tabela $\mathrm{V}$ mostra teores de $\mathrm{Fe}$ e $\mathrm{Al}$ das amostras de óxidos determinadas pela técnica de EDS. Valores de \% átomo do elemento oxigênio foram desconsiderados, devido à influência do ar ambiente e/ou porta-amostras no resultado. Comparando-se as razões $\mathrm{Fe} / \mathrm{Fe}+\mathrm{Al}$ obtidas experimentalmente, verificou-se de maneira geral que os valores encontrados foram muito próximos aos valores teóricos esperados, com exceção das amostras M e R; nestes dois casos o valor obtido foi consideravelmente abaixo do esperado. Os teores de substituições de $\mathrm{Fe}^{3+}$ por $\mathrm{Al}^{3+}$ na estrutura da hematita nessas amostras foram de $51 \mathrm{e}$ $61 \%$, respectivamente, sendo que possivelmente parte das espécies de hidróxido de ferro foi solubilizada durante a mistura dos géis ou durante a lavagem devido à composição das mesmas e/ou a efeitos sinérgicos. De maneira geral, verificou-se que o $\mathrm{pH}$ de síntese não apresentou efeito significativo no que diz respeito aos teores de $\mathrm{Fe}^{3+}$ e $\mathrm{Al}^{3+}$ presentes nas fases de hematita substituídas. Verificou-se a presença de teores de sulfato, o qual deve estar presente em poros dos materiais, bordas dos cristais ou pode ter sido formada uma fase não detectada pela técnica de DRX, possivelmente pelo baixo teor de enxofre encontrado nas amostras e/ou baixa cristalinidade da fase.

A técnica de colorimetria fornece dados que servem de parâmetros para determinação da coloração das amostras; estes parâmetros estão divididos em luminosidade ( $\left.\mathrm{L}^{*}\right)$, tendência da cor do verde ao vermelho ( $\left.\mathrm{a}^{*}\right)$, tendência da cor do amarelo ao azul ( $\left.b^{*}\right)$ e o ângulo Hue (h), o qual indica a tonalidade e a cromaticidade da cor $\left(\mathrm{C}^{*}\right)$. Os valores obtidos para cada parâmetro das amostras são mostrados na Tabela VI. Em relação às amostras de hidróxido contendo $100 \%$ de

Tabela V - Composição das amostras de óxido determinadas pela técnica de EDS.

[Table V - Composition of the oxide samples determined by the EDS technique.]

\begin{tabular}{ccccccc}
\hline Amostra & $\mathrm{pH}$ & $\begin{array}{c}\mathrm{Fe} \\
(\% \text { átomo })\end{array}$ & $\begin{array}{c}\mathrm{Al} \\
(\% \text { átomo })\end{array}$ & $\begin{array}{c}\mathrm{S} \\
(\% \text { átomo })\end{array}$ & $\begin{array}{c}\mathrm{Fe} / \mathrm{Fe}+\mathrm{Al} \\
\text { experimental }\end{array}$ & $\begin{array}{c}\mathrm{Fe} / \mathrm{Fe}+\mathrm{Al} \\
\text { teórico }^{\mathrm{a}}\end{array}$ \\
\hline $\mathrm{K}$ & 98,8 & $*$ & 1,2 & 1,0 & 1,0 \\
$\mathrm{~L}$ & & 81,3 & 17,5 & 1,2 & 0,7 & 0,7 \\
$\mathrm{M}$ & 6 & 65,6 & 32,6 & 1,8 & 0,5 & 0,6 \\
$\mathrm{~N}$ & & 62,9 & 35,7 & 1,9 & 0,5 & 0,5 \\
$\mathrm{O}$ & $*$ & 94,5 & 5,5 & 0,0 & 0,0 \\
\hline $\mathrm{P}$ & & 100,0 & $*$ & $*$ & 1,0 & 1,0 \\
$\mathrm{Q}$ & & 81,6 & 18,4 & $*$ & 0,7 & 0,7 \\
$\mathrm{R}$ & 8 & 56,6 & 42,4 & 1,0 & 0,4 & 0,6 \\
$\mathrm{~S}$ & & 66,6 & 32,5 & 0,9 & 0,5 & 0,5 \\
$\mathrm{~T}$ & $*$ & 100,0 & $*$ & 0,0 & 0,0 \\
\hline Notas: * teor não detectado; ${ }^{a}$ valores esperados em relação às quantidades de reagentes empregados na sintese.
\end{tabular}


$\mathrm{Al}$ (E e J), estas apresentaram um comportamento distinto ao observado para as amostras compostas por Fe (A e F). Nas amostras E e J observaram-se diferenças significativas em todos os valores dos parâmetros ópticos medidos, sendo que a amostra $\mathbf{J}$ apresentou maior tendência para cores claras (mais branco); isso se deve possivelmente à estrutura melhor organizada para o hidróxido de alumínio obtido em pH 8, como observado por DRX (Fig. 1b). Em geral as amostras de hidróxidos mistos apresentaram parâmetros próximos, comparando-se as amostras semelhantes em teores $\mathrm{Fe} / \mathrm{Fe}+\mathrm{Al}$, mas diferenciando no $\mathrm{pH}$ de síntese (B e $\mathrm{G} ; \mathrm{C}$ e $\mathrm{H}$; D e I). Nas amostras $\mathrm{O}$ e $\mathrm{T}$ dos óxidos contendo apenas alumínio, notou-se que os parâmetros de luminosidade ficaram próximos entre si, sendo possível observar que com o aumento do $\mathrm{pH}$ a amostra $\mathrm{T}$ apresentou uma

Tabela VI - Valores dos parâmetros colorimétricos dos hidróxidos e óxidos mistos de ferro e alumínio (Fe:Al) obtidos em pH 6 e 8 - sistema CIELAB.

[Table VI - Values of colorimetric parameters of mixed iron and aluminum ( $\mathrm{Fe}: \mathrm{Al}$ ) hydroxides and oxides obtained at $\mathrm{pH}$ 6 and 8 - CIELAB system.]

\begin{tabular}{cccccc}
\hline Amostra & $\mathrm{L}^{*}$ & $\mathrm{a}^{*}$ & $\mathrm{~b}^{*}$ & $\mathrm{C}^{*}$ & $\mathrm{~h}$ \\
\hline $\mathrm{A}$ & 34,85 & 6,63 & 8,28 & 10,60 & 54,34 \\
$\mathrm{~B}$ & 33,75 & 5,25 & 5,79 & 7,82 & 47,78 \\
$\mathrm{C}$ & 40,62 & 9,96 & 15,60 & 18,51 & 57,45 \\
$\mathrm{D}$ & 37,59 & 9,42 & 12,79 & 15,89 & 53,63 \\
$\mathrm{E}$ & 38,65 & $-0,39$ & $-9,03$ & 0,39 & 184,88 \\
$\mathrm{~F}$ & 34,54 & 6,45 & 6,70 & 9,30 & 46,07 \\
$\mathrm{G}$ & 35,12 & 8,96 & 9,90 & 13,35 & 47,85 \\
$\mathrm{H}$ & 39,81 & 8,35 & 12,57 & 15,09 & 56,40 \\
$\mathrm{I}$ & 37,36 & 8,56 & 10,20 & 13,32 & 49,99 \\
$\mathrm{~J}$ & 61,34 & $-0,92$ & 1,40 & 1,68 & 123,26 \\
$\mathrm{~K}$ & 31,68 & 5,22 & 3,18 & 6,11 & 31,31 \\
$\mathrm{~L}$ & 31,98 & 6,00 & 4,31 & 7,38 & 35,67 \\
$\mathrm{M}$ & 35,46 & 13,33 & 10,44 & 16,93 & 38,07 \\
$\mathrm{~N}$ & 33,03 & 9,12 & 6,72 & 11,33 & 36,39 \\
$\mathrm{O}$ & 63,05 & 2,58 & 6,07 & 6,59 & 67,00 \\
$\mathrm{P}$ & 31,14 & 5,74 & 3,36 & 6,65 & 30,32 \\
$\mathrm{Q}$ & 33,92 & 9,63 & 6,77 & 11,77 & 35,11 \\
$\mathrm{R}$ & 34,32 & 10,11 & 6,96 & 12,27 & 34,53 \\
$\mathrm{~S}$ & 33,31 & 9,03 & 6,75 & 11,27 & 36,79 \\
$\mathrm{~T}$ & 61,44 & $-0,62$ & 1,47 & 1,60 & 112,84 \\
\hline
\end{tabular}

luminosidade mais intensa em comparação à amostra $\mathrm{O}$, no entanto os fatores $\mathrm{a}^{*}, \mathrm{~b}^{*}, \mathrm{C}^{*} \mathrm{e} \mathrm{h}$ apresentaram mudanças significativas; isso se deve novamente à organização estrutural semelhante entre as amostras, como observado por DRX. A amostras L, M, N, P, Q, R e S apresentaram parâmetros diferentes entre si, mesmo com o aumento do $\mathrm{pH}$. Comparando-se os resultados dos parâmetros colorimétricos dos hidróxidos e óxidos puros de ferro (amostras A, F, K e P) da Tabela VI com valores reportados na literatura, este trabalho se destaca por apresentar valores distintos aos já publicados [5]. É conhecido na literatura que hematitas contendo substituições isomórficas por alumínio não apresentam influência nos parâmetros colorimétricos, apenas no brilho dos materiais, o que não foi observado neste trabalho. Os resultados obtidos indicaram que o método de síntese empregado, condições experimentais avaliadas e as propriedades estruturais obtidas influenciam nas propriedades colorimétricas, o que possibilitou a obtenção de novos materiais de baixo custo, distintos aos já conhecidos na literatura e possíveis de serem utilizados como pigmentos coloridos.

A partir dos dados anteriormente discutidos, pôde-se determinar a diferença total de cor $(\Delta \mathrm{E})$ de acordo com a Eq. A, para os hidróxidos e óxidos mistos em relação ao padrão. Foi estabelecido como padrões para comparação as amostras de hidróxido e óxido contendo $100 \%$ de ferro (amostras A, F, K e P), pois estas apresentaram coloração vermelha intensa, característica da hematita, enquanto que espécies de alumínio são brancas; dessa maneira, buscouse verificar o efeito da adição de alumínio em relação à hematita pura. Os resultados para as comparações são mostrados na Tabela VII. Observou-se de maneira geral para os hidróxidos e óxidos mistos que o aumento de $\mathrm{pH}$ de preparo dos materiais não influenciou significativamente na diferença de cor, pois os valores calculados se encontraram próximos. Coincidentemente as amostras que apresentaram maiores diferenças de cor foram aquelas que apresentaram menores parâmetros de rede, como observado por DRX. Levando em consideração os hidróxidos, as amostras C e $\mathrm{H}$ se destacaram nas diferenças de cor, assim como, entre as amostras de hidróxidos mistos, apresentaram maior cristalinidade quando caracterizadas por DRX (Fig. 1). Os óxidos derivados destas amostras também se destacaram pelas diferenças de cor e novamente foram as amostras que apresentaram diferenças estruturais que mais se destacaram comparando-se com as outras amostras, pois nas amostras

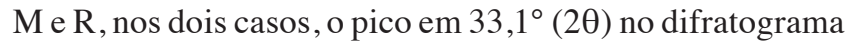
de raios $\mathrm{X}$ se apresentou mais intenso em comparação ao pico em $35,7^{\circ}(2 \theta)$, devido possivelmente às substituições isomórficas de $\mathrm{Fe}^{3+}$ por $\mathrm{Al}^{3+}$. Tal observação possivelmente

Tabela VII - Diferença total da cor da amostra em relação ao padrão pH 6 e pH 8 para os hidróxidos e óxidos. [Table VII - Total color difference of the sample relative to the standard $\mathrm{pH} 6$ and pH 8 for hydroxides and oxides.]

\begin{tabular}{ccccccccccccc}
\hline Amostras & A e B & A e C & A e D & F e G & F e H & F e I & K e L & Ke M & Ke N & Pe Q & Pe R & Pe S \\
\hline$\Delta$ Eab & 3,051 & $9,897^{*}$ & 5,969 & 4,108 & $8,114^{*}$ & 4,965 & 1,405 & $11,523^{*}$ & 5,437 & 5,873 & $6,494^{*}$ & 5,120 \\
\hline
\end{tabular}

* indica amostras que apresentaram maiores diferenças de cor. 
contribuiu para as diferenças de coloração dos materiais.

\section{CONCLUSÕES}

Foram obtidos hidróxidos/óxidos mistos de ferro-III e alumínio; as estruturas obtidas foram baseadas na hematita, sendo que as técnicas de DRX e FTIR indicaram que os sítios de ferro na estrutura foram substituídos por alumínio. O método empregado consistiu na mistura das fases de hidróxido estáveis, onde não era esperada a formação das fases mistas, porém nas condições empregadas ocorreu reorganização estrutural obtendo-se fases mistas. Os materiais foram avaliados em relação às propriedades de cor, sendo estas quantificadas pelo sistema CIELAB. Foi verificado que a adição de alumínio proporcionou diferenças nas propriedades ópticas relacionadas à cor, sendo que essas diferenças se devem, além da presença deste elemento, às propriedades estruturais dos materiais. Este trabalho se destaca pela apresentação de uma modificação de metodologia para produção de hidróxidos/óxidos de ferroIII e alumínio, onde foi possível obter materiais de baixo custo e de fácil preparo que podem ser aplicados à produção de pigmentos cerâmicos.

\section{AGRADECIMENTOS}

Os autores agradecem às instituições de ensino superior Universidade Tecnológica Federal do Paraná (UTFPR) campus Pato Branco e Universidade Estadual do CentroOeste campus CEDETEG pela estrutura física, e à CAPES pela bolsa de estudo de pós-doutorado.

\section{REFERÊNCIAS}

[1] E.L. Crepaldi, J.B. Valim, Quím. Nova 21, 3 (1997) 300. [2] D. Li, Y. Ding, X. Wei, Y. Xiao, L. Jiang, Appl. Catal. A General 507 (2015) 130.

[3] F.J. Anaissi, J.C. Villalba, S.T. Fujiwara, L.F. Cótica, C.R.L. Souza, P. Zamora-Peralta, Quím. Nova 32, 8 (2009)
2006.

[4] L.C.A. Oliveira, J.D. Fabris, M.C. Perreira, Quím. Nova 36, 1 (2013) 123.

[5] R.M. Cornell, U. Schwertmann, The iron oxides: structure, properties, reactions, occurrences and uses, $2^{\text {nd }}$ Ed., Wiley-VCH, Weinheim (2003).

[6] N.M. Ahmed, M.M. Selim, Pigm. Resin Technol. 34, 5 (2005) 256.

[7] P.M. Bertsch, W.P. Miller, M.A. Anderson, L.W. Zelazny, Clays Clay Miner. 37, 1 (1989) 12.

[8] V.M. Belousov, V.M. Chertov, É.V. Rozhkova, V.I. Litvin, V.A. Zazhigalov, Theor. Exp. Chem. 33, 2 (1997) 103.

[9] J. Ding, Q. Zhong, S. Zhang, W. Cai, J. Hazard. Mater. 283 (2015) 633-642.

[10] V.I. Mikhailov, T.P. Maslennikova, P.V. Krivoshapkin, Glass Phys. Chem. 40 (2014) 650.

[11] X. Bokhimi, J. Sánchez-Valente, F. Pedraza, J. Solid State Chem. 166 (2002) 182.

[12] Konica Minolta Sensing Americas, "Entendendo o espaço de cor $\mathrm{L}^{*} \mathrm{a}^{*} \mathrm{~b} * "$, ac. 30/11/2016.

[13] P.L. Younger, S.A. Banwart, S.R. Hedin, Mine water hydrology, pollution, remediation, $2^{\text {nd }}$ Ed., Kluwer Acad. Publ., Boston (2002) 319.

[14] M.F. Bini, "Preparação e caracterização de compostos Fe-Al-O pelo processo sol-gel", Diss. Mestr., Un. Est. Centro-Oeste, Guarapuava (2009).

[15] R.G. Gast, E.R. Landa, G.W. Meyer, Clays Clay Miner. 22 (1974) 31.

[16] A.B. Kiss, G. Keresztury L. Farkas, Spectrochim. Acta A 36 (1980) 653.

[17] S. Tresintsi, K. Simeonidis, N. Pliatsikas, G. Vourlias, P. Patsalas, M. Mitrakas, J. Solid State Chem. 213 (2014) 145.

[18] T. Basu, K. Gupta, U.C. Ghosh, Chem. Eng. J. 183 (2012) 303.

[19] Y. Wang, F. Li, S. Dong, X. Liu, M. Li, J. Colloid Interface Sci. 467 (2016) 28.

(Rec.03/12/2016, Rev. 11/02/2017, 14/06/2017, 13/10/2017, Ac. 04/01/2018) 